\title{
Social Connections and Access Charges in Networks
}

\author{
Rodrigo Harrison ${ }^{1}$, Gonzalo Hernandez ${ }^{2}$, and Roberto Munoz ${ }^{3}$ \\ 1 P.U.C. de Chile, Instituto de Economia \\ 2 UNAB, Escuela de Ingenieria Civil and \\ U. de Chile, Centro de Modelamiento Matematico \\ ${ }^{3}$ CIDE Mexico, Division de Economia and \\ U.T.F.S.M. Chile, Departamento de Industrias
}

\begin{abstract}
In this paper we present a model where two interconnected network operators compete in linear prices in a market characterized by the existence of social connections among consumers, which are represented by a random regular graph. Assuming horizontal differentiation among operators, the customers select their network provider based on their preferences and the prices offered by the competing firms. In equilibrium the number of calls made to other agents depends on where they are located in the social network.
\end{abstract}

\section{Introduction}

The study of network interconnection problems has been a very active area of research over the last decade. One of the main reasons for the interest in this topic is its immediate impact in terms of regulatory policy, especially in telecommunication markets 1 The literature has studied the case where competing networks need to be connected to their rivals in order to provide a service. In such a case, the access charge can play a key role in terms of the equilibrium arising in a competitive framework. For example, [7] developed a model where, in the presence of linear and nondiscriminatory pricing schemes to consumers, the access charge can be used by the firms as a collusive device. That is, the firms can agree to pay each other high access charges because their profits are maximized in this way. However, consumer and social surplus are reduced, consequently, regulatory intervention is called for. However, a key assumption has not been scrutinized enough. They assumed a balanced calling pattern, meaning that "the percentage of calls originated on a network and completed on the same network is equal to the fraction of consumers subscribing to this network". In order to represent the assumption in a simple way, they assume that demand is formed by a continuous of individuals where each of them connects (or makes calls to) other individuals with the same intensity if they are confronted with the same price of the service.

\footnotetext{
${ }^{1}$ See [1] for a survey.
} 
In a mobile phone market, for example, this modeling approach implies that each person, confronted to equal prices, would make the same number of calls to their relatives and friends than to any other unrelated person in the market. This unrealistic assumption is usually made for simplicity, because it permits to characterize market shares for the firms in a very simple way. More importantly, the assumption permits to characterize the equilibrium behavior of the players using closed form expressions that facilitate the comparative static. However, to the best of our knowledge, no attempt has been made to study the robustness of the results of these models to the presence of such a strong behavioral assumption.

In this paper we depart from the traditional approach by abandoning the assumption of the existence of a continuous of consumers connecting to each other with the same intensity. Instead, we present a model where two interconnected network operators compete in linear and non discriminatory prices in a market characterized by the existence of social connections among consumers, which are modeled through a social network. The balanced calling pattern is no longer an assumption, but it can arise in equilibrium 2 As usual, each operator has to pay an access charge to its rival when the services demanded by its consumers have to be completed in the rival network. Assuming horizontal differentiation among operators 3 the customers select their preferred network based on their tastes and the prices offered by the competing firms. The main difference with previous studies is that we recognize that an individual in the market belongs to a social network 4 therefore, if he or she makes a call to an individual placed closer in the network then he or she receives a higher utility than from a call to an individual located farther away, even if the price for the service is the same. As a consequence, in equilibrium the number of calls made to others depends on where they are located in the social network.

The rest of the paper is organized as follows: In section 2 we develop the economic model, including the agent's demand, the firms' problem and the game played by the two firms. In section 3 we obtain numerical results when the social network is modeled using random regular graphs. In section 4 the conclusions are stated.

\section{The Economic Model}

The model assumes the existence of a social network, represented by a graph $g$. Nodes in the graph represent agents (indexed by $i \in I$ ) and the links show how people are interconnected socially. There are two firms, $A$ and $B$, offering comunication services (i.e. two cellular companies) and individuals have to decide which firm to subscribe to. In order to make the affiliation decision, agents take

${ }^{2}$ See [3] and [4 for results considering heterogenous agents.

${ }^{3}$ Horizontal differentiation means that the services offered by the competing networks are not different in quality, thereby consumer preferences determine affiliation decisions.

${ }^{4}$ See [5] and [6] for further details on network theory and economics. 
into account the prict5 per call offered by each firm, $p_{A}$ and $p_{B}$, and his or her taste. Taste is modeled in a standard way: each agent $i$ in the social network (i.e. each node in $g$ ) is endowed with a realization of a taste random variable $x_{i}$, from a cumulative density function $F$ with support in $[0,1]$. In what follows we assume that firm $A$ is "located" in 0 and firm $B$ in 1 . None of them provide the "ideal service" to agent $i$, positioned in $x_{i}$ (this would be the case if some network were located precisely in $x_{i}$ ). Introducing a unit transportation cost of $t$, the cost of selecting a service different from $i$ 's ideal one is $t x_{i}$ if agent $i$ selects network $A$ or $t\left(1-x_{i}\right)$ if network $B$ is preferred.

\subsection{The Agent Demand}

Consider the affiliation decision problem of agent $i$. If agent $i$ decides to connect to network $j=A, B$ then we will say that he/she belongs to the set $I_{j}$.

Agent $i$ 's demand for calls is represented by the vector $q_{i}=\left(q_{i j}\right)_{j \in g, j \neq i}$, where the generic element $q_{i j}$ is the number of calls that agent $i$ makes to agent $j$. Then the gross utility of agent $i$ can be described as follows:

$$
U_{i}\left(q_{i}\right)=\sum_{j \in g, j \neq i} \lambda_{i} \delta^{t_{i j}} u\left(q_{i j}\right) \quad \text { with } \quad u\left(q_{i j}\right)=\frac{q_{i j}^{1-1 / \eta}}{1-1 / \eta}
$$

where:

$\delta$ : is a discount factor, that is $0<\delta<1$.

$t_{i j}$ : is the shortest distance (in terms of links) connecting agents $i$ and $j$. The more distant is $j$ from $i$ in the social network, the lower is the utility of a call. If they are not connected then $t_{i j}=\infty$.

$\eta$ : is a constant parameter representing the elasticity of demand.

$\lambda_{i}$ : scale factor associated with the total amount of social connections of agent $i 6$

Now let us start assuming that $i$ decides to connect to firm $A$. Given that agent $i$ observes the price of network $A, p_{A}$, he/she is going to select the vector of calls $q_{i}=\left(q_{i j}\right)_{j \in g, j \neq i}$ to all his contacts in the network $g$ so that:

$$
V_{i}\left(p_{A}\right)=\max _{q_{i}}\left\{\lambda_{i} U_{i}\left(q_{i}\right)-p_{A} \sum_{j \in g, j \neq i} q_{i j}\right\}
$$

Solving this maximization problem, we obtain his/her demand's components:

$$
q_{i j}\left(p_{A}\right)=\left(\frac{p_{A}}{\lambda_{i} \delta^{t i j}}\right)^{-\eta}
$$

\footnotetext{
${ }^{5}$ We just consider linear and non dicriminatory prices.

${ }^{6}$ In the simulation we will use the scaling parameter $\lambda_{i}=\left(\delta C\left(g_{i}\right)^{\frac{1}{\eta}}\right)^{-1}$, where $C\left(g_{i}\right)$ stands for the total amount of connections of the agent.
} 
Intuitively, for the same price $p_{A}$, agent $i$ makes more calls to contacts located closer in the social network $g$ than farther in it. Therefore, plugging into equation 2 we get the indirect utility function:

$$
V_{i}\left(p_{A}\right)=\sum_{j \in g, j \neq i} \lambda_{i}^{\eta} \delta^{\eta t_{i j}}{\frac{p_{A}}{\eta-1}}^{1-\eta}
$$

and an analogous result arise for firm $B$.

It is easy to see that each agent, given his/her taste parameter, will select the network providing him/her the highest indirect utility net of transportation costs. Agent $i$ 's decision of affiliation will depend on his/her taste parameter value $x_{i}$ in the following way: there is an $x_{i}^{*}$ representing an indifferent value, such that if $x_{i}<x_{i}^{*}\left(\operatorname{resp} . x_{i}>x_{i}^{*}\right)$ he/she will join network $A$ (resp. $\left.B\right)$. Such $x_{i}^{*}$ is defined by:

$$
V_{i}\left(p_{A}\right)-x_{i}^{*} t=V_{i}\left(p_{B}\right)-\left(1-x_{i}^{*}\right) t
$$

Solving for $x_{i}^{*} 7$ we get:

$$
x_{i}^{*}=\frac{1}{2}+\sigma \frac{\left(p_{A}^{1-\eta}-p_{B}^{1-\eta}\right)}{\eta-1} \sum_{j \in g, j \neq i} \lambda_{i}^{\eta} \delta^{\eta t_{i j}} \quad \text { with } \quad \sigma=\frac{1}{2 t}
$$

\subsection{The Firm's Problem}

Assuming that each firm pursues the maximization of its profits and access charges are given, then firm $A$ (resp. $B$ ) will select its price $p_{A}$ (resp. $p_{B}$ ) such that:

$$
\begin{aligned}
& \max _{p_{A}} \pi_{A}\left(p_{A}, p_{B}\right)= \\
& \sum_{i \in I_{A}}\left\{\sum_{\substack{j \neq i \\
j \in I_{A}}} q_{i j}\left(p_{A}\right)\left(p_{A}-c_{A}^{o}-c_{A}^{f}\right)+\sum_{j \in I_{B}} q_{i j}\left(p_{A}\right)\left(p_{A}-c_{A}^{o}-a_{B}\right)-f\right\}+ \\
& \sum_{i \in I_{B}} \sum_{j \in I_{A}} q_{i j}\left(p_{B}\right)\left(a_{A}-c_{A}^{f}\right)
\end{aligned}
$$

where 8

$f$ : is the fixed cost incurred by a firm when it affiliates a new subscriber. $c_{A}^{o}$ : is the cost of originating a call for firm $A$ ( $c_{B}^{o}$ is defined analogously).

$c_{A}^{f}$ : is the cost of terminating a call for firm $A$ ( $c_{B}^{f}$ is defined analogously). $a_{A}$ : is the price firm $A$ charges firm $B$ in order to terminate a call from a subscriber of $B$ to a subscriber of $A$ ( $a_{B}$ is defined analogously).

\footnotetext{
${ }^{7}$ It is important to note that $x_{i}^{*}$ does not depend on where $i$ 's contacts are affiliated. This is not the case when networks are permitted to use different linear prices schemes for own and rival subscribers (the discriminatory case).

${ }^{8}$ In what follows, when we solve an optimization problem, we assume that $g, f, c_{A}^{o}$, $c_{B}^{o}, c_{A}^{f}, c_{B}^{f},\left\{x_{i}\right\}_{i=1}^{I}, t$ are all given exogenously.
} 
Using the previous definitions, the problem for firm $A$ becomes:

$$
\begin{aligned}
\max _{p_{A}} \pi_{A}\left(p_{A}, p_{B}\right)= & \left(p_{A}-c_{A}^{o}-c_{A}^{f}\right) p_{A}^{-\eta} \sum_{i \in I_{A}} \sum_{\substack{j \neq i \\
j \in I_{A}}} \delta^{\eta t_{i j}}+ \\
& \left(p_{A}-c_{A}^{o}-a_{B}\right) p_{A}^{-\eta} \sum_{i \in I_{A}} \sum_{j \in I_{B}} \delta^{\eta t_{i j}}- \\
& \sum_{i \in I_{A}} f+\left(a_{A}-c_{A}^{f}\right) p_{B}^{-\eta} \sum_{i \in I_{B}} \sum_{j \in I_{A}} \delta^{\eta t_{i j}}
\end{aligned}
$$

The problem for firm $B$ is analogous.

\subsection{The Game}

We are interested in a Subgame Perfect Nash Equilibrium (SPNE) of a two stage game played by firms. We analyze two cases.

In the first case, the unregulated case, firms are free to select noncooperatively their access charges, $a_{A}$ and $a_{B}$, in a first stage and then, in the second stage, they set noncooperatively the prices to consumers $p_{A}^{*}$ and $p_{B}^{*}$.

In the second case, the regulated case, the access charges for both firms are determined exogenously in the first stage (by a regulator) as equal to the marginal costs $\left(a_{A}=a_{B}=c_{A}^{f}=c_{B}^{f}\right)$. In the second stage the firms set noncooperatively the prices to consumers $p_{A}^{*}$ and $p_{B}^{*}$.

In both cases we are interested in the equilibrium outcome and how it is modified when some parameters change. It is also in our interest to measure consumer surplus, so given equilibrium prices $\left(p_{A}^{*}, p_{B}^{*}\right)$ in any of the cases described above, we evaluate:

$$
C S\left(p_{A}^{*}, p_{B}^{*}\right)=\sum_{i \in I_{A}} V_{i}\left(p_{A}^{*}\right)+\sum_{i \in I_{B}} V_{i}\left(p_{B}^{*}\right)-t\left[\sum_{i \in I_{A}} x_{i}+\sum_{i \in I_{B}}\left(1-x_{i}\right)\right]
$$

\section{$3 \quad$ Numerical Results}

The numerical study was performed using medium scale simulations. The social network was modeled by random regular graphs of degree $d$, see [2]. The access costs and prices varied within the predefined range: $\left[a_{\min }, a_{\max }\right],\left[p_{\min }, p_{\max }\right]$. The goal of the simulations was to compare the outcomes of the regulated and unregulated cases. The methodology for the simulations in the unregulated case was the following 9

0) Define parameters: $I, d, \sigma, \eta, \delta, c_{A}^{o}, c_{B}^{o}, c_{A}^{f}, c_{B}^{f}$ and $f$

1) Generate random graph of degree $d$.

2) Generate random vector of network preferences: $x=\left(x_{i}\right)_{i \in I}$

\footnotetext{
${ }^{9}$ The methodology in the regulated case is trivially implied.
} 
3) For each access charge profile $\left(a_{A}, a_{B}\right)$ in $\left[a_{\min }, a_{\max }\right] \times\left[a_{\min }, a_{\max }\right]$ we solve the second stage of the game:

(a) For each price profile $\left(p_{A}, p_{B}\right)$ in $\left[p_{\min }, p_{\max }\right] \times\left[p_{\min }, p_{\max }\right]$ :

i) Compute indifference values: $x^{*}=\left(x_{i}^{*}\right)_{i \in I}$

ii) Compute the utility functions of firms $A$ and $B$ :

$\pi_{A}\left(p_{A}, p_{B}\right), \pi_{B}\left(p_{A}, p_{B}\right)$

(b) Compute price response functions:

$r_{A}\left(p_{A}, p_{B}\right), r_{B}\left(p_{A}, p_{B}\right)$

(c) Compute SPNE price reaction functions in the second stage:

$p_{A}^{*}\left(a_{A}, a_{B}\right), p_{B}^{*}\left(a_{A}, a_{B}\right)$

4) Going back to the first stage, compute access cost response functions $r_{a_{A}}\left(a_{A}, a_{B}\right), r_{a_{B}}\left(a_{A}, a_{B}\right)$

5) Compute SPNE access charges in the first stage $a_{A}^{*}, a_{B}^{*}$ and evaluate price reaction functions at these values: $p_{A}^{*}\left(a_{A}^{*}, a_{B}^{*}\right), p_{B}^{*}\left(a_{A}^{*}, a_{B}^{*}\right)$

6) Evaluate indifference values at the equilibrium outcome:

$x^{*}\left(a_{A}^{*}, a_{B}^{*}\right)=\left(x_{i}^{*}\left(p_{A}^{*}\left(a_{A}^{*}, a_{B}^{*}\right), p_{B}^{*}\left(a_{A}^{*}, a_{B}^{*}\right)\right)\right)_{i \in I}$

7) Evaluate firms' benefits equilibrium outcome:

$\pi_{A}\left(p_{A}^{*}\left(a_{A}^{*}, a_{B}^{*}\right), p_{B}^{*}\left(a_{A}^{*}, a_{B}^{*}\right)\right), \pi_{B}\left(p_{A}^{*}\left(a_{A}^{*}, a_{B}^{*}\right), p_{B}^{*}\left(a_{A}^{*}, a_{B}^{*}\right)\right)$

8) Evaluate consumer surplus $C S\left(p_{A}^{*}\left(a_{A}^{*}, a_{B}^{*}\right), p_{B}^{*}\left(a_{A}^{*}, a_{B}^{*}\right)\right)$ as in eq. 10

9) Repeat steps (1) to (8) for $T$ different graphs keeping parameters defined in step $(0)$.

The size of the social network was fixed at $I=1024$ and random regular graphs with degrees $d=10,15,20$ were used. The access charge discretization selected 5 values: 2 above and 2 below the regulated case. The price discretization considered 20 equidistant values. Table 1 below contains average results for simulations. The rest of the parameters were fixed as follows: $\sigma=1, \eta=1.5$, $\delta=0.9, c_{A}^{o}=c_{B}^{o}=0.75, c_{A}^{f}=c_{B}^{f}=0.75$ and $f=0.5$.

Table 1. Numerical results when $d$ is modified

\begin{tabular}{|c|c|c|c|c|c|c|c|}
\multicolumn{1}{c}{} & \multicolumn{4}{c}{ Firm A } & \multicolumn{3}{c|}{ Firm B } \\
\cline { 2 - 9 } \multicolumn{1}{c|}{} & d & regulated & unreg. & change \% & regulated & unreg. & change \% \\
\hline \multirow{3}{*}{ access charge } & 10 & 0.75 & 1.05 & 40 & 0.75 & 1.05 & 40 \\
\cline { 2 - 8 } & 15 & 0.75 & 1.05 & 40 & 0.75 & 1.05 & 40 \\
\cline { 2 - 9 } & 20 & 0.75 & 1.05 & 40 & 0.75 & 1.05 & 40 \\
\hline \multirow{3}{*}{ prices } & 10 & 3.10 & 3.45 & 11.3 & 3.15 & 3.50 & 11.1 \\
\cline { 2 - 9 } & 15 & 3.05 & 3.40 & 11.5 & 3.10 & 3.20 & 3.2 \\
\cline { 2 - 9 } & 20 & 3.05 & 3.40 & 11.5 & 3.10 & 3.20 & 3.2 \\
\hline \multirow{3}{*}{ utilities } & 10 & 77958 & 83428 & 7.1 & 77223 & 83010 & 7.5 \\
\cline { 2 - 8 } & 12 & 82417 & 83439 & 1.2 & 81931 & 87951 & 7.4 \\
\cline { 2 - 8 } & 20 & 84905 & 85892 & 1.1 & 84400 & 90658 & 7.4 \\
\hline
\end{tabular}

\begin{tabular}{|c|c|c|c|c}
\cline { 2 - 5 } \multicolumn{1}{c|}{} & d & regulated & unregulated & change $\%$ \\
\hline \multirow{4}{c}{ consumer surplus } & 10 & 709771 & 672630 & -5.2 \\
\cline { 2 - 5 } & 15 & 765856 & 742197 & -3.0 \\
\cline { 2 - 5 } & 20 & 788867 & 764515 & -3.0 \\
\hline
\end{tabular}




\section{Conclusions}

Our preliminary results show that an eventual deregulation in the determination of access charges would have a big impact on the level of access charges but a relatively minor impact on prices, utilities and consumer surplus. On the other hand, an increase in the connectivity of the social network (implying an increase in demand keeping constant the number of individuals forming the network) has a minor impact over access charges and prices, ceteris paribus the regulatory environment. However, firms' benefits and consumer surplus increase significantly, especially in the regulated and unregulated case respectively, closing the gap between both regulatory frameworks. Overall the simulations show that deregulation can be an attractive alternative in front of a costly regulation, especially when the social network presents a high level of connectivity. This result contrasts with the standard recommendations in the literature for the interconnection problem under linear and nondiscriminatory pricing schemes.

\section{References}

1. Armstrong, M.: The Theory of Access Pricing and Interconnection, Handbook of Telecommunications Economics, vol. 1 (2002), Edited by Martin Cave et al. Amsterdam: North-Holland.

2. Bollobas, B., Random Graphs, Cambridge University Press (2001).

3. Dessein, W.: Network Competition with Heterogeneous Customers and Calling Patterns, Information Economics and Policy, vol. 16 (2004).

4. Hahn, J.: Network Competition and Interconnection with Heterogeneous Subscribers, International Journal of Industrial Organization vol. 22 (2004).

5. Jackson, M.: The Stability and Efficiency of Economic and Social Networks, mimeo (2001) Caltech.

6. Jackson, M. and A. Wolinsky: A Strategic Model of Social and Economic Networks, Journal of Economic Theory, vol. 71 (1996).

7. Laffont, J., P. Rey and J. Tirole: Network Competition: I. Overview and Nondiscriminatory Pricing, RAND Journal of Economics, vol. 29, (1998).

8. Laffont, J., P. Rey and J. Tirole: Network Competition: II. Price Discrimination, RAND Journal of Economics, vol. 29 (1998). 